\title{
Diagnosis and management of inhibitors: the need for professional collaboration
}

\section{Peter Collins}

Advances in the understanding of haemophilia require effective collaboration, both at the national and international levels. Such collaborations are currently playing a major role in elucidating the natural history of acquired haemophilia, and also in clarifying the issue of product-related inhibitor development in previously untreated patients with haemophilia.

Keywords: inhibitor, haemophilia, Katharine Dormandy

The naming of the Arthur Bloom haemophilia centre in Cardiff and the Katharine Dormandy haemophilia centres at the Royal Free Hospital after those who founded them provides an historical memory. Arthur Bloom and Katharine Dormandy were of a similar age, and it is inevitable that they spent time discussing haemophilia care with each other. It is difficult to overstate the vision of such people in setting up multidisciplinary haemophilia centres, the model which we now call comprehensive care centres. This model of care has become a standard for many chronic diseases. In addition, these visionary founders pulled together haemophilia clinicians from around the country into a cohesive, UK-wide network. The collaborative approach they engendered continues to inform practice internationally through research and guidelines.

\section{Acquired haemophilia}

The European Acquired Haemophilia Registry (EACH2) is an example of international collaboration involving 501 patients from 90 centres in 12 countries [1-3]. The Royal Free Hospital has enrolled 12 patients into this study, which has generated a number of influential publications. Acquired haemophilia is a bleeding disorder caused by an auto-antibody to factor VIII, which results in severe bleeding in the soft tissue and mucosa and is associated with a high morbidity and mortality. The factor VIII level is a relatively poor guide to bleeding risk, the reason for this is not well understood and remains to be elucidated through research.

Acquired haemophilia is frequently associated with diagnostic and treatment delay because patients typically present to non-specialists. In EACH2, the median time from abnormal bleeding to an abnormal aPTT is one day, which is acceptable, while the median time from an abnormal aPTT to diagnosis is also one day. However,

Peter Collins, Arthur Bloom Haemophilia Centre, School of Medicine, Cardiff University, University Hospital of Wales, Cardiff, CF14 4XN, UK Email: peter.collins@wales.nhs.uk

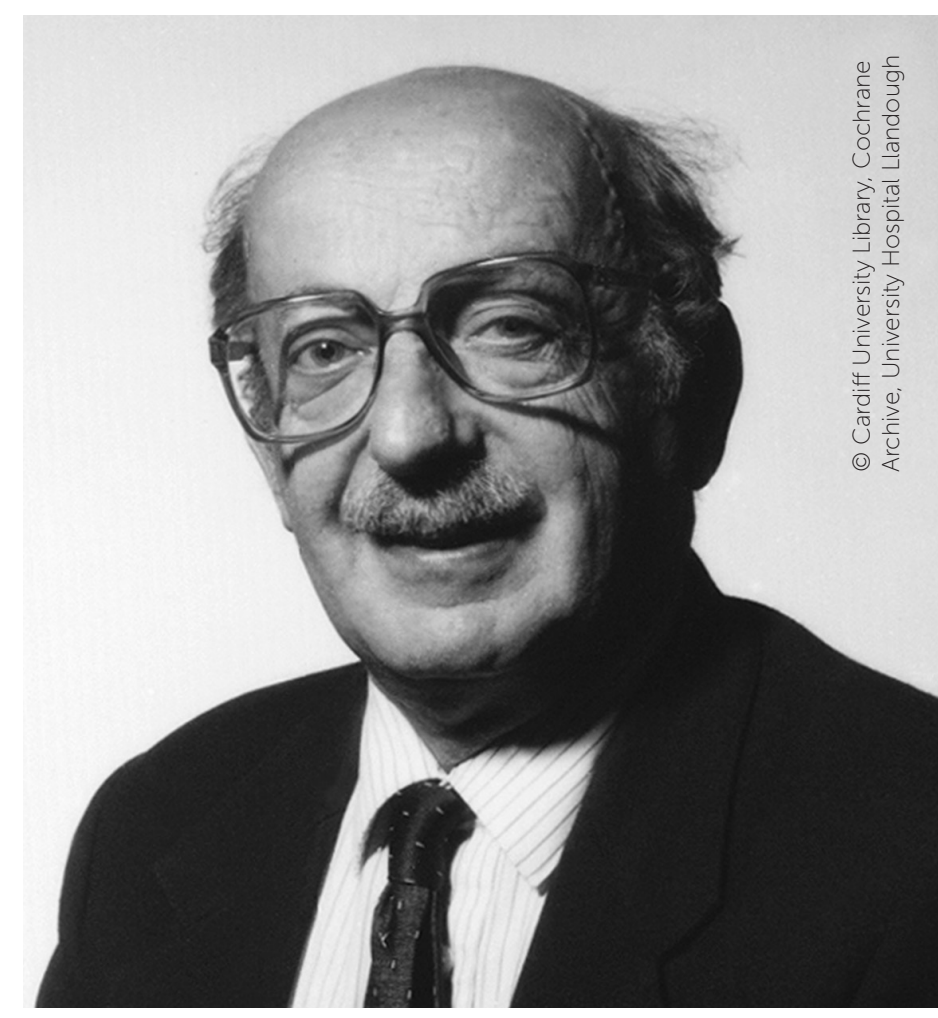

Like Katharine Dormandy, Arthur Bloom (right) also recognised the power of multidisciplinary care and collaboration in haemophilia

for $25 \%$ of patients, there is a delay of seven days until laboratory testing; and for $11 \%$ of patients there is a delay of more than 30 days, which is unacceptable given the severity of acquired haemophilia [4]. It is an important role for laboratory staff when they receive a clotting screen to investigate abnormalities, interpret the result and ensure that the clinicians understand the meaning of results. Data from the UK acquired haemophilia study show that fatal bleeding can occur as long as three or four months after presentation if the inhibitor is not eradicated. It also shows that a measureable factor VIII level and lack of bleeding at presentation does not protect against fatal bleeding. Data from the registry show that inhibitor eradication was better achieved by a combination of steroids and cyclophosphamide rather than just steroids alone. although the study wasn't observational and not randomised. Treatment with rituximab alone was associated with slower remission, as is suggested by several studies in the literature. However, first-line of 
treatment has no effect on survival at one year This information is important in developing new clinical studies.

Once a diagnosis has been made there are good options for treatment of bleeding in acquired haemophilia and the main challenge is to reduce the toxicity associated with immunosuppression. In the EACH2 registry, 3\% of people died of bleeding but more than $10 \%$ died of the effect of immunosuppression.

\section{Inhibitors and product-related factors}

Recent national and international collaborations have sought to investigate the issue of product-related inhibitor development in previously untreated patients with haemophilia. Data from the RODIN study suggested a potential association between inhibitor formation and a recombinant factor VIII product [5]. The study involved specialised referral centres and all patients could not enrolled because of referral practices. When the RODIN study was published the UKHCDO Inhibitor working party decided to investigate further using the National Haemophilia Database, one of the most important components of UK haemophilia care. All previously untreated patients in the UK were identified in the time span that mirrored the RODIN study [6]. All patients first treated in the UK were included irrespective of where they were initially treated and continued to be followed if they changed centre. This resulted in a consecutive cohort, from which it was possible to identify all those who developed inhibitors. Among all patients, there was a $29 \%$ inhibitor rate as reported in previous studies. Furthermore, those patients who were excluded had a $29 \%$ inhibitor rate. Patients were excluded if they were first treated outside the UK (it could not be certain what product these patients had been treated with), those who had more than one brand of factor VIII in the first reporting period (it was not be possible to be sure what their first treatment was). In addition, four children had died in the first year of life and had to be excluded

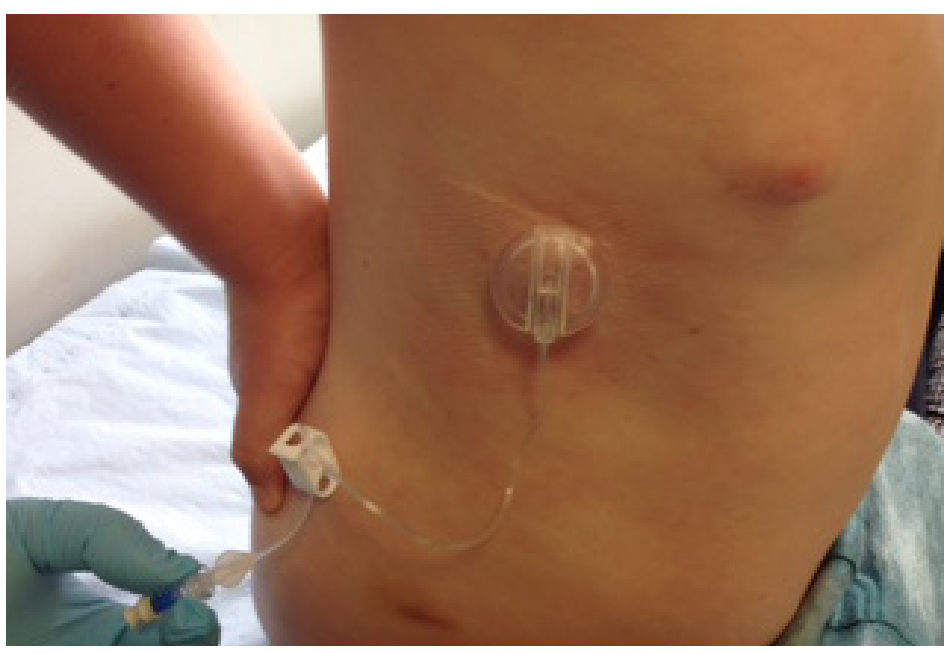

Greater use of port-a-caths across the UK in recent years may have impacted upon the study findings (because they had not had time to be exposed to factor VIII and develop inhibitors) while those treated with firstgeneration Helixate $(n=2)$ were also excluded.

Analysis of the risk factors in the UK previously untreated patients confirmed previously described risk factors. For high titer inhibitors, the adjusted hazard ratio was significantly increased in the non-white population, but this was only of borderline significance for all inhibitors. The strongest risk factor identified was genotype, the high risk mutations being associated with both high titer and all inhibitors. Intensive treatment at first exposure was associated with all inhibitors; there was no association with high titer inhibitors and there was no association with family history of haemophilia or the age of first exposure.

The results regarding factor VIII product and inhibitor risk were similar to the RODIN findings showing an increased risk of inhibitors with Kogenate Bayer. However, given that this study was done because of the RODIN study and some of the UK patients had been included in RODIN. Therefore the analysis was repeated excluding the UK RODIN patients and when this was done there was a borderline result for high titer inhibitors.

The strength of this analysis is that it is based on a completely consecutive cohort of UK patients and it was possible to include patients on clinical trials. It is very important that within the UK database it is possible to record what concentrates people are on, particularly as more patients are now going into clinical trials. It may be that at some stage in the future there will be a potential issu with a new concentrate that is now in a trial, and it may be important to be able to look back and repeat this sort of study within the national database.

The UK study involved high quality data entry which took almost a year to complete and some of larger centres had to do a huge amount of work to provide the data. But in the end, it was possible to look at plausible inhibitor numbers and risk factors. However, the study is not randomised, which is the major shortcoming in both the UK and the RODIN data. Subconsciously, clinicians may have giving Kogenate to people who had a higher risk of developing an inhibitor. Also, the UK data do not include data on exposure days (time is used as a surrogate for exposure), and the concentrates used changed over the study time. This is particularly important as the proportion of patients on any given product changed over time, as new products come to the market and as a result of the national tender. Treatment practices may also have changed over the time of the study, with more early intensive exposure, with more inhibitor surveillance and greater use of port-a-caths. It may be that the study is showing a time effect. There was an attempt to adjust the data for year of first treatment in the analysis; while this did not change the analysis, it is not quite sufficient to look for the effect of time.

Another factor that should be considered is centreeffect. There was an adjustment for this and no difference emerged but it cannot definitely be ruled out. In terms of time, splitting the data into three cohorts suggested that the 


\section{Peter Collins}

Peter Collins is a professor of haematology at the Institute of Infection and Immunity School of Medicine of Cardiff University and an honorary consultant haematologist and director of the Arthur Bloom Haemophilia Centre at the University Hospital of Wales. He worked as a lecturer at the Katharine Dormandy Haemophilia Centre for two years. The experience convinced him that he wished to spend his career looking after people with inherited bleeding disorders. His time at the Royal Free also taught him of the importance of comprehensive care delivered by a multidisciplinary team, the synergistic importance of combining clinical care with research and the importance of patient involvement at all levels. He is now chair of the National Institutes of Health Research UK Haematology Specialty Group and chair of the UKHCDO Inhibitor Working Party. His research interests include acquired haemophilia, prophylactic treatment in haemophilia, factor VIII inhibitors, haemostatic impairment during postpartum haemorrhage and the role of phospholipids in controlling coagulation.

increased risk of inhibitors with Kogenate was seen in the first and second cohort, but in the third the reverse was true.

The paper concluded that it could not definitely be shown that there is an increased risk of inhibitors with Kogenate, but that the data were being put into the public domain to inform the debate. The European Medicines Agency is now re-analysing all the data from the UK and the French study [7] along, with the RODIN data, and a new opinion will come out of that. UKHCDO is now working closely with the EMA to make that data available.

\section{Disclosures}

The scientific meeting on which this issue of The Journal of Haemophilia Practice is based was sponsored by Baxter, Bayer, CSL Behring, Grifols, Novo Nordisk, Sobi, Pfizer, BPL and Werfen. Editorial support for the article was provided by the publisher.

\section{References}

1. Knoebl P, Marco P, Baudo F, et al. Demographic and clinical data in acquired hemophilia A: results from the European Acquired Haemophilia Registry (EACH2). J Thromb Haemost 2012; 10(4): 622-31. doi: 10.1111/j.1538-7836.2012.04654.x. 2. Baudo F, Collins $P$, Huth-Kühne A, et al. Management of bleeding in acquired hemophilia A: results from the European Acquired Haemophilia (EACH2) Registry. Blood 2012; 120(1): 39-46. doi: 10.1182/blood-2012-02-408930.

3. Collins P, Baudo F, Knoebl P, et al. Immunosuppression for acquired hemophilia A: results from the European Acquired Haemophilia Registry (EACH2). Blood 2012; 120(1): 47-55. doi: 10.1182/blood-2012-02-409185

4. Tengborn L, Baudo F, Huth-Kühne A, et al. Pregnancy-associated acquired haemophilia A: results from the European Acquired Haemophilia $(E A C H 2)$ registry. BJOG 2012; 119(12): 1529-37. doi: 10.1111/j.1471-0528.2012.03469.x.

5. van den Berg HM, Gouw SC, van der Bom JG, et al. Factor VIII products and inhibitors in severe hemophilia A. N Engl J Med 2013; 368(15): 1457. doi: 10.1056/ NEJMc1301995

6. Collins PW, Palmer BP, Chalmers EA, et al. Factor VIII brand and the incidence of factor VIII inhibitors in previously untreated UK children with severe hemophilia A, 2000-2011. Blood 2014; 124(23): 3389-97. doi: 10.1182/blood-2014-07-580498. 7. Calvez T, Chambost H, Claeyssens-Donadel S, et al. Recombinant factor VIII products and inhibitor development in previously untreated boys with severe hemophilia A. Blood 2014; 124(23): 3398-408. doi: 10.1182/blood-2014-07-586347.

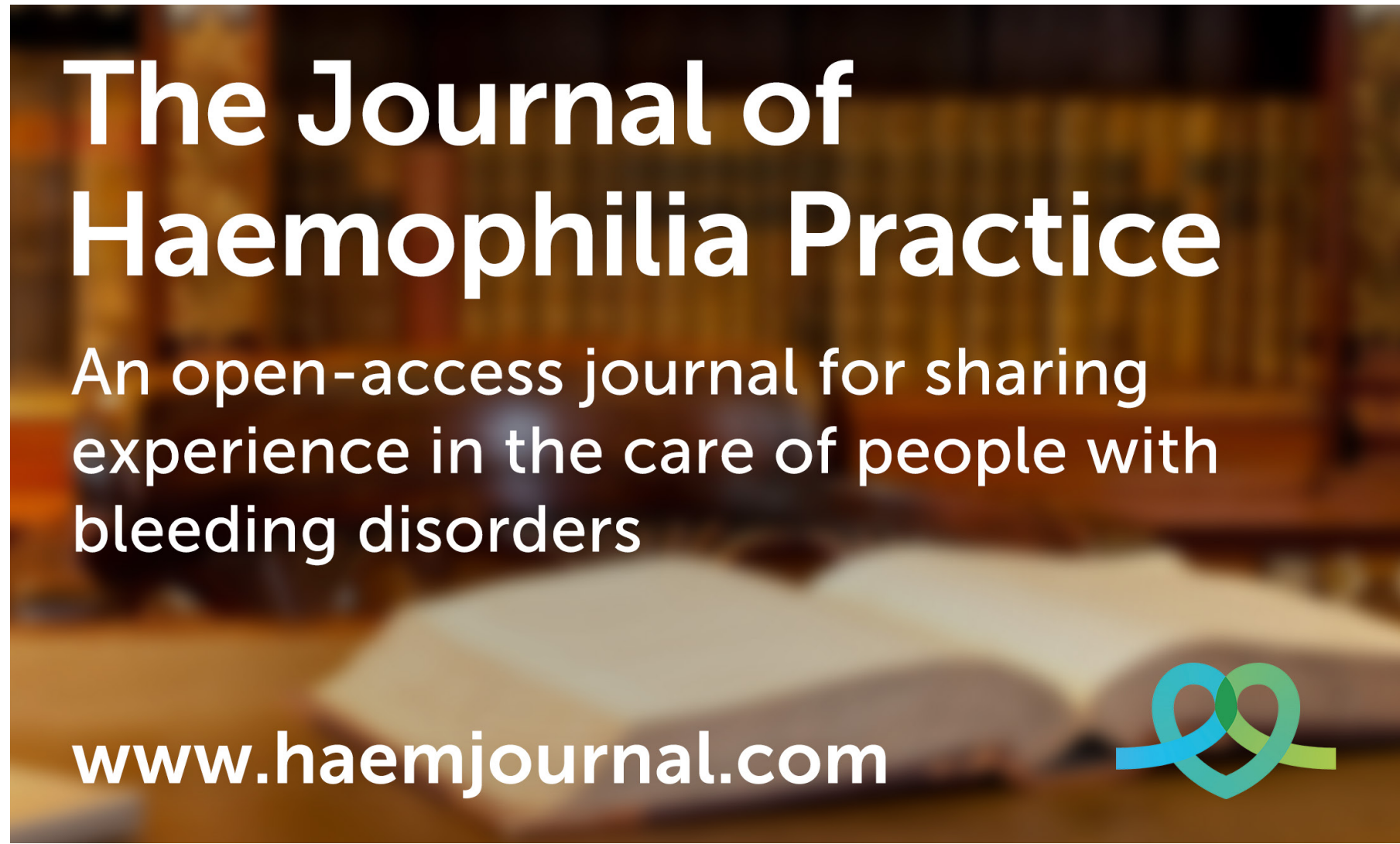

